\title{
Systemic anaplastic large cell lymphoma presenting as a huge mediastinal mass in a case of hyper lgE syndrome: a case report
}

\author{
Atefeh Kheyrollahiyan ${ }^{1 \dagger}$, Akbar Sharifi $^{2+}$ and Mohammad Mirza-Aghazadeh-Attari ${ }^{3,4^{*}}$ (D)
}

\begin{abstract}
Background: Hyper IgE syndrome is a rare heterogeneous immunodeficiency syndrome which is characterized by recurrent episodes of cutaneous and respiratory tract infections and development of cold abscesses. This syndrome is also associated with malignancies, most notability hematologic malignancies.

Case presentation: In this case report, we discuss a 27-year-old male with proven hyper lgE syndrome and effected by tuberculosis, who developed an anaplastic large cell lymphoma, a very rare and aggressive subtype of lymphoma. We will discuss imaging findings in this rare case. The case presented here had a large mediastinal mass, which had encircled the aorta, and was accompanied by subcarinal lymphadenopathy and inguinal lymphadenopathy.

Conclusions: Systemic anaplastic large cell lymphoma is a rare subtype of lymphoma which is rarely associated with hyper lgE syndrome. In this case, both lymphoma and tuberculosis infection were witnessed in the same patient, showing a classic example of immune dysregulation.
\end{abstract}

Keywords: CT scan, Hyper IgE syndrome, Lymphoma, Anaplastic large cell lymphoma, Tuberculosis

\section{Background}

Hyper immunoglobulinemia E (IgE) syndrome is a heterogeneous group of disorders characterized by immune dysregulation. Davis et al. [1] reported the first case of hyper IgE syndrome in 1966, in two girls with chronic dermatitis and cold abscesses, and named the condition "Job's syndrome." Later, it was discovered that indeed Job's syndrome was a subtype of hyper IgE syndrome, with an autosomal dominant form of transmission. To some extent, all forms of hyper IgE syndrome present with findings such as chronic dermatitis, recurrent cutaneous infections, chronic sinusitis, pneumatoceles, atopy, and increased rate of certain malignancies, most notably lymphomas. Immunologically, this condition is accompanied by reduced IL-22, IL-21, IL-11, IL-10, and IL-17 signaling; reduced chemotaxis of neutrophils; and

\footnotetext{
* Correspondence: m.aghazadeh75@yahoo.com

${ }^{+}$Atefeh Kheyrollahiyan and Akbar Sharifi contributed equally to the study.

${ }^{3}$ Medical Radiation Sciences Research Group, Faculty of Medicine, Tabriz University of Medical Sciences, Tabriz, Iran

${ }^{4}$ Aging Research Institute, Tabriz University of Medical Sciences, Tabriz, Iran Full list of author information is available at the end of the article
}

abnormal differentiation of B lymphocytes [2]. Because of the latter, patients suffering from hyper IgE syndrome are prone to aggressive lymphomas. In this case report, we present a patient with documented hyper IgE syndrome who suffered from recurrent infections in the respiratory tract and the skin, and had been diagnosed and was being treated for tuberculosis. This patient developed an anaplastic lymphoma during follow-up, which presented as a huge mediastinal mass.

\section{Case presentation}

A 27-year-old male presented with a chief complaint of cough exacerbation within the previous month.

He was a known case of hyper IgE Syndrome for 17 years with a history of recurrent pneumonia, chronic sinusitis, skin rashes, nail infections, coarse texture of the skin, and delayed dental shedding.

He had a family history of a similar condition in his elder brother and sister, causing an early death in both siblings because of sepsis. The medical records of both siblings were unfortunately not available, but genetic 


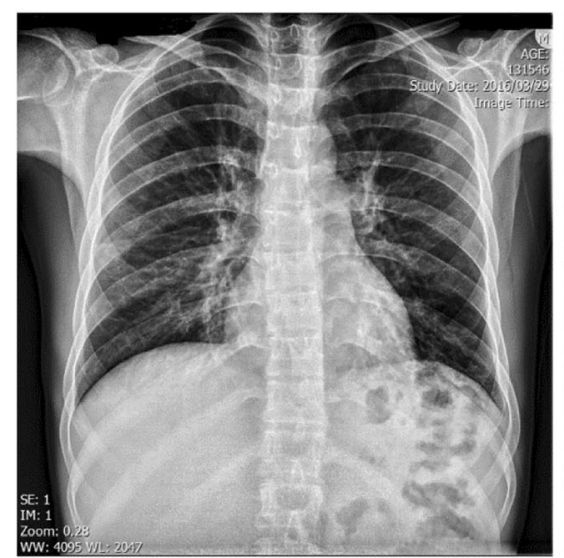

A

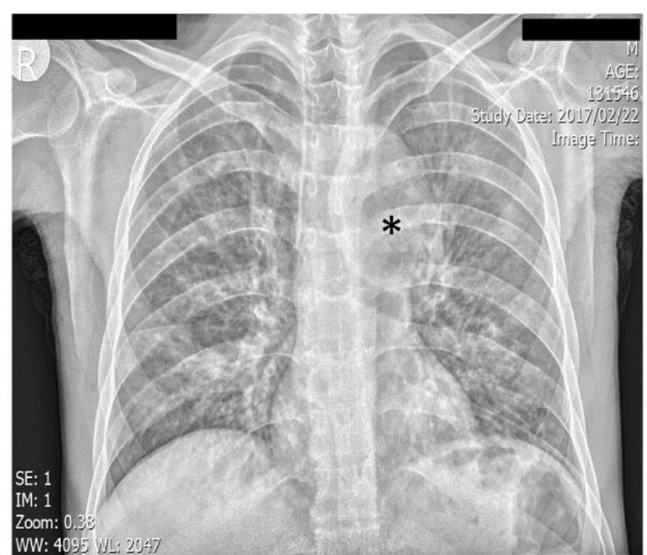

B

Fig. 1 Two chest X-rays of the patient taken 1 year apart. a The chest X-ray taken when the patient was diagnosed with tuberculosis. $\mathbf{b}$ The chest $\mathrm{X}$-ray taken when the patient had exacerbated symptoms after 1 year. A giant mass is seen in the mediastinum, which was not present before.

The asterisk marks the described lesion

analysis of the patient had revealed a mutation in the STAT3 gene.

From childhood, as the disease had begun, the patient experienced recurrent episodes of respiratory tract infections and rashes on the skin. The lesions were superinfected, and the patient was on medication with antistaphylococcus agents, which had resulted in moderation of the skin lesions.

He had lost light perception in his right eye when he was 12 years old due to cutaneous lesions and their superimposed infections.

A year ago, he was admitted to a pulmonary subspecialty ward because of pneumonia (with fever and chills, productive coughs, and yellow-brown sputum), was diagnosed with tuberculosis during clinical examination, and received the classic TB treatment, comprising of rifampin, isoniazid, ethambutol, and pyrazinamide (10-15 mg/ $\mathrm{kg}$ of each medication).

Two months after starting the anti-TB medications, due to high liver functional enzymes (SGOT $=89$ to $>77$ to $>$ 135, SGPT $=99$ to $>101$ to $>77$ ) and a high ALK.P (4847 to $>4175$ to $>991$ ) in a number of lab tests, all TB medications were halted and the patient underwent further evaluation.

He underwent abdominal and pelvic CT scan which showed an enlarged liver (liver span in longest dimension equaled $20 \mathrm{~cm}$ ) with 2 hypodense masses with a maximum diameter of $17 \mathrm{~mm}$. Numerous large hypodense masses in both inguinal regions suggestive of lymphadenopathy and a mass-like lesion with a diameter of $41 \times 77 \mathrm{~mm}$ in LLQ were found. The patient chose

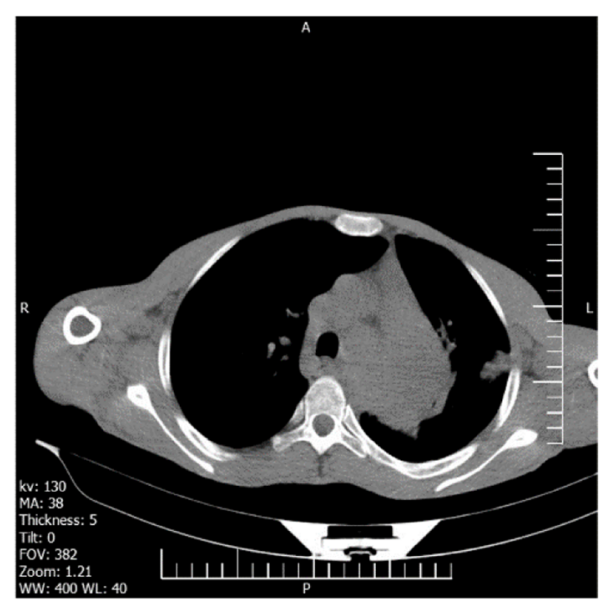

A

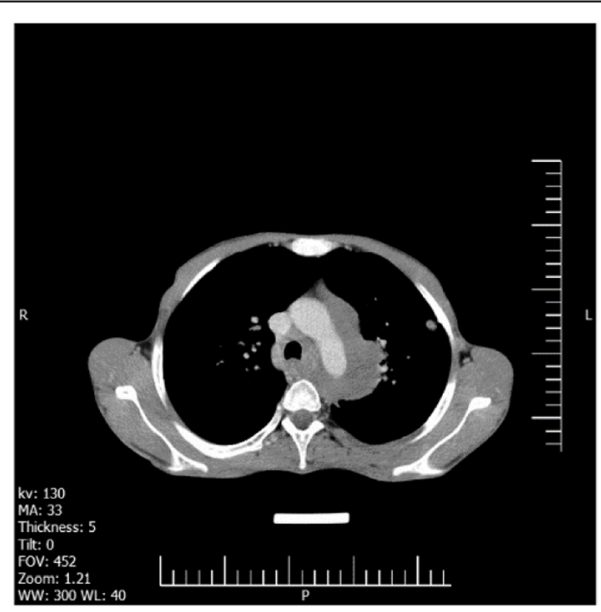

B

Fig. 2 CT scan of the lungs and mediastinum with and without contrast. a An enlarged mass exists in the mediastinum. $\mathbf{b}$ Venous phase of $C T$ with contrast shows that the aortic arch is encased by a mass which is not enhanced 

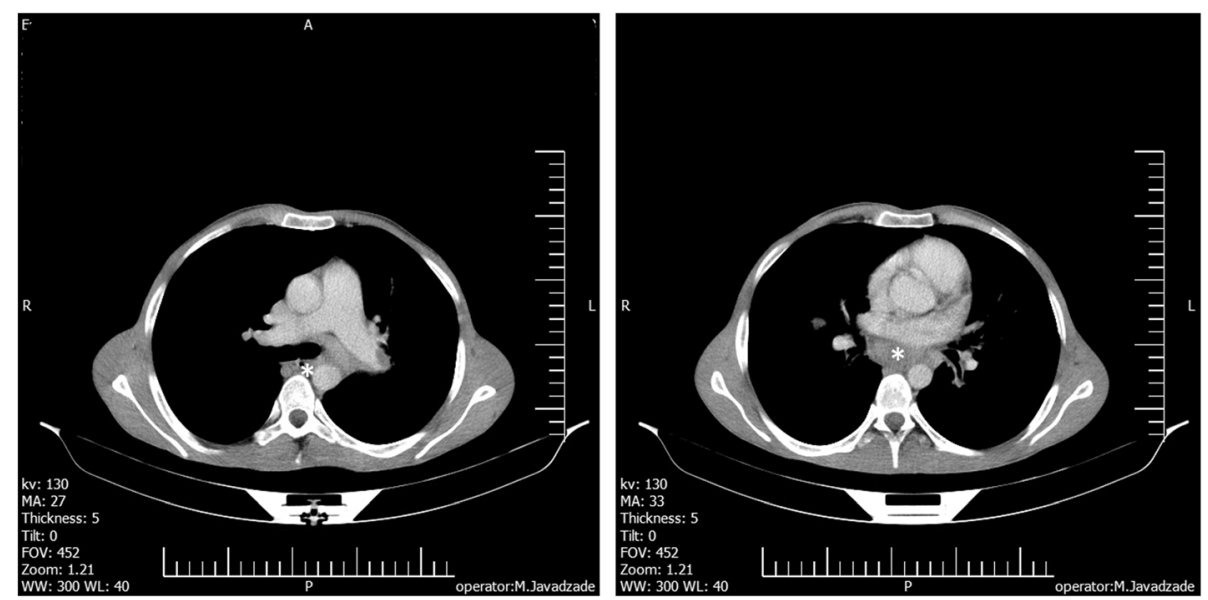

Fig. 3 Subcarinal lymphadenopathy detected by CT with venous contrast. The lymphadenopathy is shown by the asterisk

not to continue further evaluations and left the hospital on personal demand.

Twenty days prior to the last admission, the patient was admitted to the hospital because of cough exacerbation, fever, and chills. Reduced lung sounds and fine crackles were auscultated in both lung bases, and the liver was $5 \mathrm{~cm}$ below the rib cage.

He received antibiotics and supportive treatment for pulmonary infection and underwent chest radiography, which showed a huge mass in the left thoracic cavity, just above the heart. Because of this, the patient had a CT scan with intravenous contrast, which showed an infiltrative large mass of $60 \times 78 \mathrm{~mm}$ in the left paramediastinum with aortic encasement and subcarinal

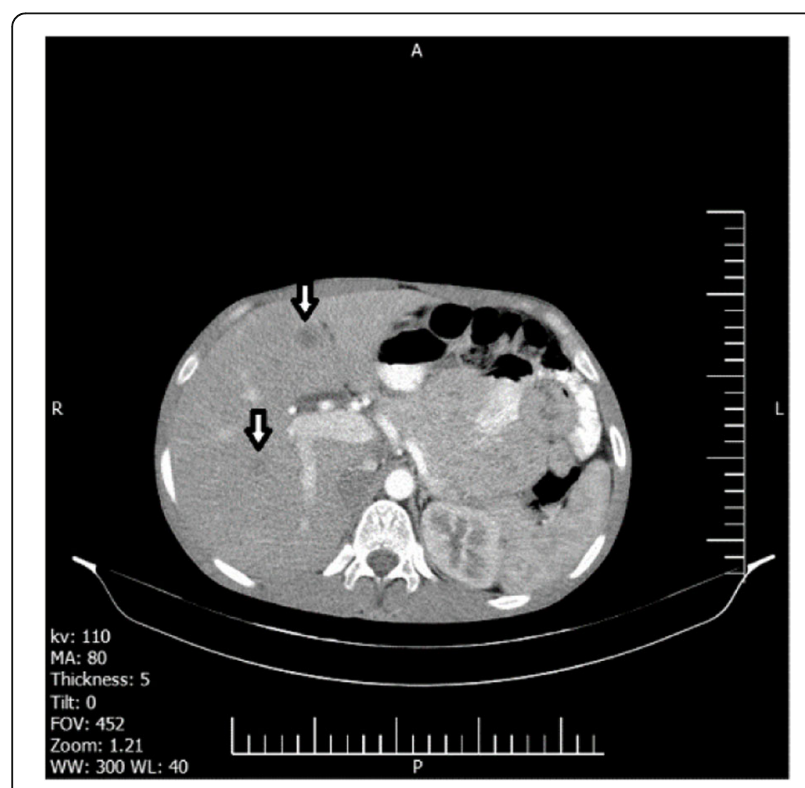

Fig. 4 Triple-contrast CT scan of the abdomen. Low attenuated lesions are seen in the liver (arrows) lymphadenopathy. Numerous nodules with a maximum diameter of about $17 \mathrm{~mm}$ were also seen in both lungs. A mosaic pattern with ground glass opacification and an increase in intralobular septum thickness were observed. A consultation with a clinical oncologist suggested biopsy of the lesions. Imaging findings of the patient are presented in Figs. 1, 2, 3, 4, 5, and 6.

Bronchial biopsy and washing were not diagnostic; therefore, open lung biopsy was performed after consultation with the thoracic surgery specialists and immunohistochemical analysis of the specimen showed strong immune reactivity of all large neoplastic cells for CD30 and their negative reaction for CD3, CD15, CD20, CD45, CK, S100, and vimentin. Numerous reactive $\mathrm{CD} 20+\mathrm{CD} 45+\mathrm{B}$ cells and many reactive $\mathrm{CD} 3+\mathrm{CD} 45+\mathrm{T}$ cells were also seen, which overall were in favor of anaplastic large cell lymphoma. Further examination revealed a positive Ki-1 marker. Further consultation with the oncology department resulted in the patient being treated with the $\mathrm{CHOP}$ regimen (cyclophosphamide, doxorubicin, vincristine, and prednisone). The patient was under treatment with this regimen for 2 months and was being considered to undergo treatment with brentuximab vedotin, until an episode of neutropenic fever caused the patient to be hospitalized in the ICU ward. The patient deteriorated swiftly and died within the first week of ICU hospitalization.

\section{Discussion}

In this article, we report a case of hyper IgE syndrome with typical dermatologic manifestations which was complicated by both a tuberculosis infection and an anaplastic large cell lymphoma. The first paramedical findings leading us to the diagnosis of lymphoma were a large mass in both chest X-rays and lung CT 


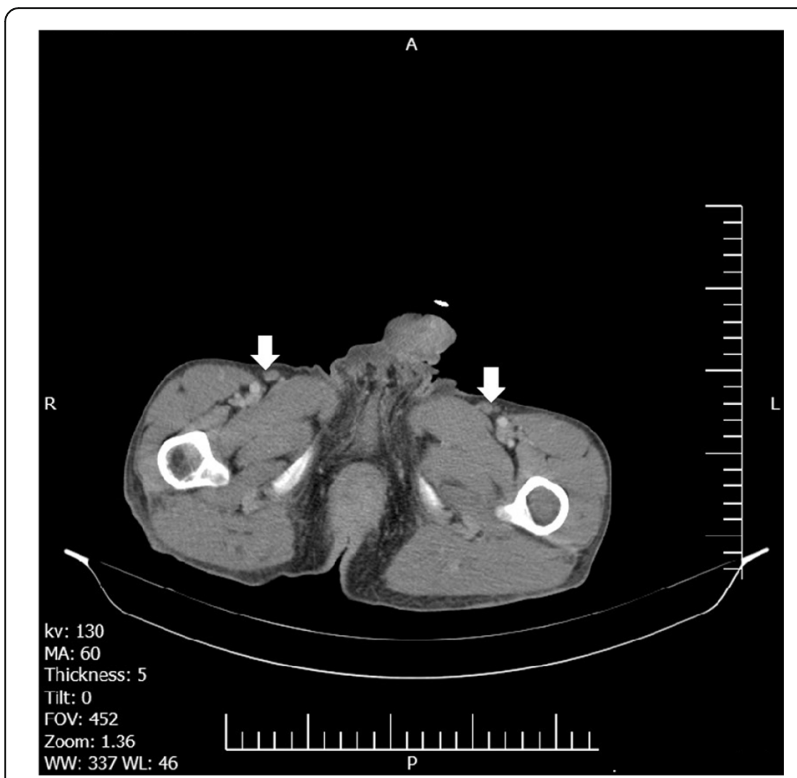

Fig. 5 Bilateral inguinal lymphadenopathy showed by the arrows

scans, which had encircled the aorta. Immunohistochemically, staining was done on a biopsy specimen of the lung, which yielded the definite diagnosis. Hyper IgE syndrome is associated with a dysfunctional immune response, which can present both as susceptibility to infectious agents and autoimmune diseases. Importantly, clinicians have observed that hyper IgE syndrome is associated with Hodgkin's and non-Hodgkin's lymphoma, especially the autosomal dominant subtype, which is associated with STAT3 deficiency [3]. Interestingly, both pro-inflammatory and anti-inflammatory responses are mediated through STAT3 signaling. Tangible evidence of this role is seen in hyper IgE syndrome, where both antiand pro-inflammatory responses are dysfunctional [4].
Another form of hyper IgE syndrome is the autosomal recessive subtype, which is caused by a deficiency in dedicator of cytokinesis 8 (DOCK8), a molecule involved in the regulation of actin, and possibly a tumor suppressor. Clinically, this subtype is not associated with connective tissue diseases, while it is mostly associated with neurologic symptoms, mucocutaneous viral infections and asthma, and food allergies, unlike the autosomal dominant type $[5,6]$.

Although these two subtypes have different clinical manifestations and pathophysiological processes, they are both associated with malignancies, including lymphomas. A number of case reports have shed light on patients with hyper IgE syndrome who have been diagnosed with lymphomas. These reports have found that most patients have suffered of either diffuse large B cell lymphomas, T cell lymphomas, Burkitt lymphomas, Mantle cell lymphoma, Hodgkin's lymphoma, or rare cases of extranodal NK/T lymphomas [7]. In this case report, we present a patient with a history of hyper IgE syndrome who was diagnosed with anaplastic large cell lymphoma, a very rare finding, of which only one has been reported so far [8], and in a rather young age (27 years old). This subtype of lymphoma is characterized by the presence of CD30 and Ki-1 tumor markers and is associated with cutaneous involvement, involvement of the skeletal system, spleen, liver, and soft tissues. The pathophysiology of this rare subtype of lymphoma is associated with the activation of anaplastic lymphoma kinase, a receptor tyrosine kinase in the insulin receptor superfamily [9]. Of interest, in vivo studies have suggested that there may be a link between anaplastic lymphoma kinase activation and STAT3, and the combined function of these two molecules may protect hematopoietic cells from apoptosis [10].
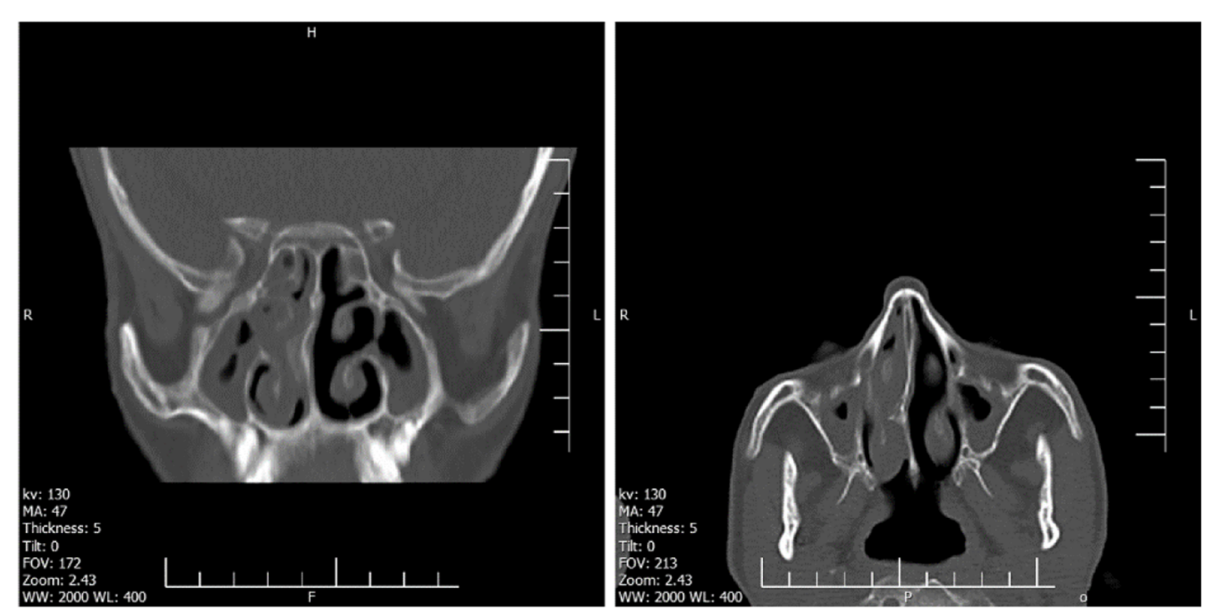

Fig. 6 Coronal and axial cuts of the sinuses. Ostiomeatal complex pattern is seen, as both the ethmoidal, maxillary, and frontal sinuses are involved 
This patient also used to suffer from tuberculosis, again a rather rare finding, as most patients with hyper IgE syndrome are complicated by grampositive bacteria. Furthermore, our case was novel in regard to imaging findings, as no other case had presented with a large mass in the mediastinum.

A general overview of previous case reports published in this regard reveals that CT scan has been the most utilized imaging method of lymphomas, and findings have included involvement of the parotid glands, spleen, and abdominal, mediastinal, and inguinal lymph nodes. Various studies have reported sizes up to $5 \mathrm{~cm}$ for individual masses, but larger ones are exceedingly rare. Positron emission tomography (PET) scan is also used in the detection of lymphoma in hyper IgE patients. This modality could be of interest in patients who cannot routinely undergo imaging with contrast media used in CT and MRI or those who have lesions which are not suitable for obtaining a biopsy [11].

\section{Conclusion}

We present a rare case of anaplastic large cell lymphoma in a young male patient who had previous infection with tuberculosis. Imaging in this patient unraveled a large mass in the mediastinum, which had encased the aorta. Further imaging showed numerous lymphadenopathies in the subcarinal and inguinal region. Open lung biopsy was done to attain a specimen for further analysis. IHC showed positive staining for CD30, and the diagnosis was made. This case report illustrates the duality of immune system dysfunction in individuals affected by hyper IgE syndrome. It is important for clinicians to address this duality in clinical practice and anticipate conditions resulting from defective immune system and also uncontrollable activation of the immune system.

\section{Abbreviations}

CD: Cluster of differentiation; CT scan: Computed tomography scan; Hyper IgE syndrome: Hyper immunoglobulin E syndrome; STAT3: Signal transducer and activator of transcription 3; TB: Tuberculosis

\section{Acknowledgements}

We would like to thank Dr. Puri for his support in conducting this report.

\section{Authors' contributions}

AK contributed to the data acquisition, manuscript preparation, and approval of the final version of the study. AS contributed to the oversight over the case report, data acquisition, and approval of the final version of the study. MMAA contributed to the manuscript preparation, revision of the manuscript and final edit, interpretation of imaging findings, and approval of the final version of the manuscript.

\section{Funding}

No one was paid during this study. The study did not have a source of funding. This study was not supported by a grant.

Availability of data and materials

All data is available based on a reasonable request.

\section{Ethics approval and consent to participate}

The present study was approved by the ethical board of the hospital in which the study was performed. The patient reported in this article had signed a written informed consent form. This case report was a reporting of a case in a medical educational center, in which all patients are informed that they may be subjects of scientific experiments and are informed of the ethical codes of conducts. This study was in compliance to the latest version of the Helsinki Declaration.

\section{Consent for publication}

The patient had written and signed an informed consent note that the findings may be published without any personal detail.

\section{Competing interests}

The authors declare that they have no competing interests.

\section{Author details}

${ }^{1}$ Connective Tissue Diseases Research Center, Tabriz University of Medical Sciences, Tabriz, Iran. ${ }^{2}$ Tuberculosis and Lung Disease Research Center, Tabriz University of Medical Sciences, Tabriz, Iran. ${ }^{3}$ Medical Radiation Sciences Research Group, Faculty of Medicine, Tabriz University of Medical Sciences, Tabriz, Iran. ${ }^{4}$ Aging Research Institute, Tabriz University of Medical Sciences, Tabriz, Iran.

Received: 12 August 2019 Accepted: 6 September 2019

Published online: 18 September 2019

\section{References}

1. Davis S, Schaller J, Wedgwood R, Harvard M (1966) Job's syndrome: recurrent, "cold", staphylococcal abscesses. Lancet. 287(7445):1013-1015

2. Mogensen TH (2013) STAT3 and the hyper-lgE syndrome: clinical presentation, genetic origin, pathogenesis, novel findings and remaining uncertainties. Jak-Stat. 2(2):e23435

3. Freeman AF, Holland SM (2008) The hyper-lgE syndromes. Immunol Allergy Clin North Am 28(2):277-291

4. Freeman AF, Holland SM (2010) Clinical manifestations of hyper lgE syndromes. Disease markers. 29(3, 4):123-130

5. Liza M, Gaurav D, Prasenjeet M, Swapna J, Binodini B (2018) Autosomalrecessive hyper-lgE syndrome. Indian J Dermatol 63(1):79

6. Chu EY, Freeman AF, Jing H, Cowen EW, Davis J, Su HC et al (2012) Cutaneous manifestations of DOCK8 deficiency syndrome. Archives of dermatology. 148(1):79-84

7. Kumánovics A, Perkins SL, Gilbert H, Cessna MH, Augustine NH, Hill HR (2010) Diffuse large B cell lymphoma in hyper-lgE syndrome due to STAT3 mutation. J Clin Immunol 30(6):886-893

8. Lee MW, Choi JH, Sung KJ, Moon KC, Koh JK (2003) Extensive xanthelasma associated with anaplastic large cell lymphoma and hyperimmunoglobulin E syndrome. Int J Dermatol 42(12):944-946

9. Webb TR, Slavish J, George RE, Look AT, Xue L, Jiang Q et al (2009) Anaplastic lymphoma kinase: role in cancer pathogenesis and smallmolecule inhibitor development for therapy. Expert Rev Anticancer Ther 9(3):331-356

10. Zamo A, Chiarle R, Piva R, Howes J, Fan Y, Chilosi M et al (2002) Anaplastic lymphoma kinase (ALK) activates Stat3 and protects hematopoietic cells from cell death. Oncogene. 21(7):1038

11. Friedberg JW, Chengazi V (2003) PET scans in the staging of lymphoma: current status. Oncologist. 8(5):438-447

\section{Publisher's Note}

Springer Nature remains neutral with regard to jurisdictional claims in published maps and institutional affiliations. 